\title{
Traumatic aortic injury score (TRAINS): an easy and simple score for early detection of traumatic aortic injuries in major trauma patients with associated blunt chest trauma
}

\author{
Victor X. Mosquera, Milagros Marini, Javier Muñiz, Vanesa Asorey-Veiga, Belen \\ Adrio-Nazar, Ricardo Boix, José M. Lopez-Perez, Gonzalo Pradas-Montilla and José J. \\ Cuenca
}

\begin{abstract}
Purpose. To develop a risk score based on physical examination and chest X-ray findings to rapidly identify major trauma patients at risk of acute traumatic aortic injury (ATAI).

Methods. A multicenter retrospective study was conducted with 640 major trauma patients with associated blunt chest trauma classified into ATAI (aortic injury) and NATAI (no aortic injury) groups. The score data set included 76 consecutive ATAI and 304 NATAI patients from a single center, whereas the validation data set included 52 consecutive ATAI and 208 NATAI patients from three independent institutions. Bivariate analysis identified variables potentially influencing the presentation of aortic injury. Confirmed variables by logistic regression were assigned a score according to their corresponding beta coefficient which was rounded to the closest integer value (14).

Results. Predictors of aortic injury included widened mediastinum, hypotension less than $90 \mathrm{mmHg}$, long bone fracture, pulmonary contusion, left scapula fracture, hemothorax, and pelvic fracture. Area under receiver operating characteristic curve was 0.96 . In the score data set, sensitivity was $93.42 \%$, specificity $85.85 \%$, Youden's index 0.79 , positive likelihood ratio 6.60 , and negative likelihood ratio 0.08 . In the validation data set, sensitivity was $92.31 \%$ and specificity $85.1 \%$.

Conclusions. Given the relative infrequency of traumatic aortic injury, which often leads to missed or delayed diagnosis, application of our score has the potential to draw necessary clinical attention to the possibility of aortic injury, thus providing the chance of a prompt specific diagnostic and therapeutic management.
\end{abstract}

Keywords: Aorta, Trauma, Imaging, Risk prediction, Acute aortic syndrome

\section{Introduction}

Acute traumatic aortic injury (ATAI) usually occurs in patients with major trauma and has devastating consequences [1]. Nowadays, the way of managing ATAI has evolved thanks to the advent of multidetector computed tomography (MDCT) [2], the clinical management with aggressive blood pressure control and cardiac contractility [3,4], the shift toward the use of aortic endovascular repair techniques [5], and the institution of delayed surgical treatment after the associated critical injuries have been stabilized [6]. Nevertheless, an important number of patients may not completely benefit from all the advances achieved in ATAI management as a result of a delay in the aortic injury diagnosis which may lead to catastrophic aortic-related complications [7]. Furthermore, the imaging diagnosis of some ATAI requires a specific arterial MDCT scan with multiplanar reconstructions (MPR) $[2,8]$. On the other hand, if every major trauma patient undergoes an arterial MDCT scan with MPR for a potential ATAI, the cost and level of radiation exposure would be prohibitive.

A combination of data from initial physical examination and on admission chest X-ray (CXR) to determine the probability of ATAI may allow the prompt establishment of a specific therapeutic management of ATAI to avoid aortic-related complications and a better determination of what imaging is appropriate and how it should be interpreted. As a tool like this is lacking, the purpose of this study was to determine whether a simple and easy score to determine a patient's probability of having an ATAI could be developed and validated and, if so, to estimate its diagnostic accuracy. 


\section{Patients and methods}

\section{Patient recruitment}

This retrospective study population included 646 major trauma patients with associated blunt chest trauma divided into two data sets: a score data set provided by one institution, and an independent validation data set provided by three other institutions. All the participating institutions are level-one trauma centers. Major trauma patients with associated blunt chest trauma were classified into ATAI (associated acute traumatic aortic injury) and NATAI (no associated acute traumatic aortic injury) groups.

For the purpose of the study, a major trauma patient was defined as a victim of trauma of sufficient energy to put him at risk of important injury, with associated blunt chest trauma, transported to a levelone trauma center presenting with an injury severity score (ISS) [9] greater than 15 according to published literature $[9,10]$.

The severity of the associated chest trauma was not itself an inclusion/exclusion criterion. Indeed, the severity of the associated blunt chest trauma could span from mild findings of chest trauma (i.e., local pain) to the most severe chest injuries such as bilateral lung contusion or multiple rib fractures and flail chest.

The score data set initially included 82 ATAI patients admitted to our institution from January 1980 to December 2010. However, six patients $(7.3 \%)$ in the ATAI group were excluded from the analysis because of deficient documentation and/or in extremis status on arrival. To achieve four control subjects in the NATAI group per patient with ATAI, we selected 324 consecutive patients who presented to our emergency department with major trauma with thoracic involvement but without traumatic aortic injury between January 2009 and December 2010.

In the validation data set, the ATAI group included 52 consecutive major trauma patients with aortic injury admitted between January 2000 and December 2010 at the emergency departments of three independent hospitals from different regions of the country. The validation data set also included 208 consecutive major trauma patients with thoracic involvement but without aortic injury who presented to these three collaborating centers' emergency departments between January 2009 and December 2010.

Penetrating trauma was an exclusion criterion in the study.

Figure 1 depicts a flow diagram describing the design of the study and the flow of patients. There was not a specific matching process for control patient selection apart of the aforementioned criteria.

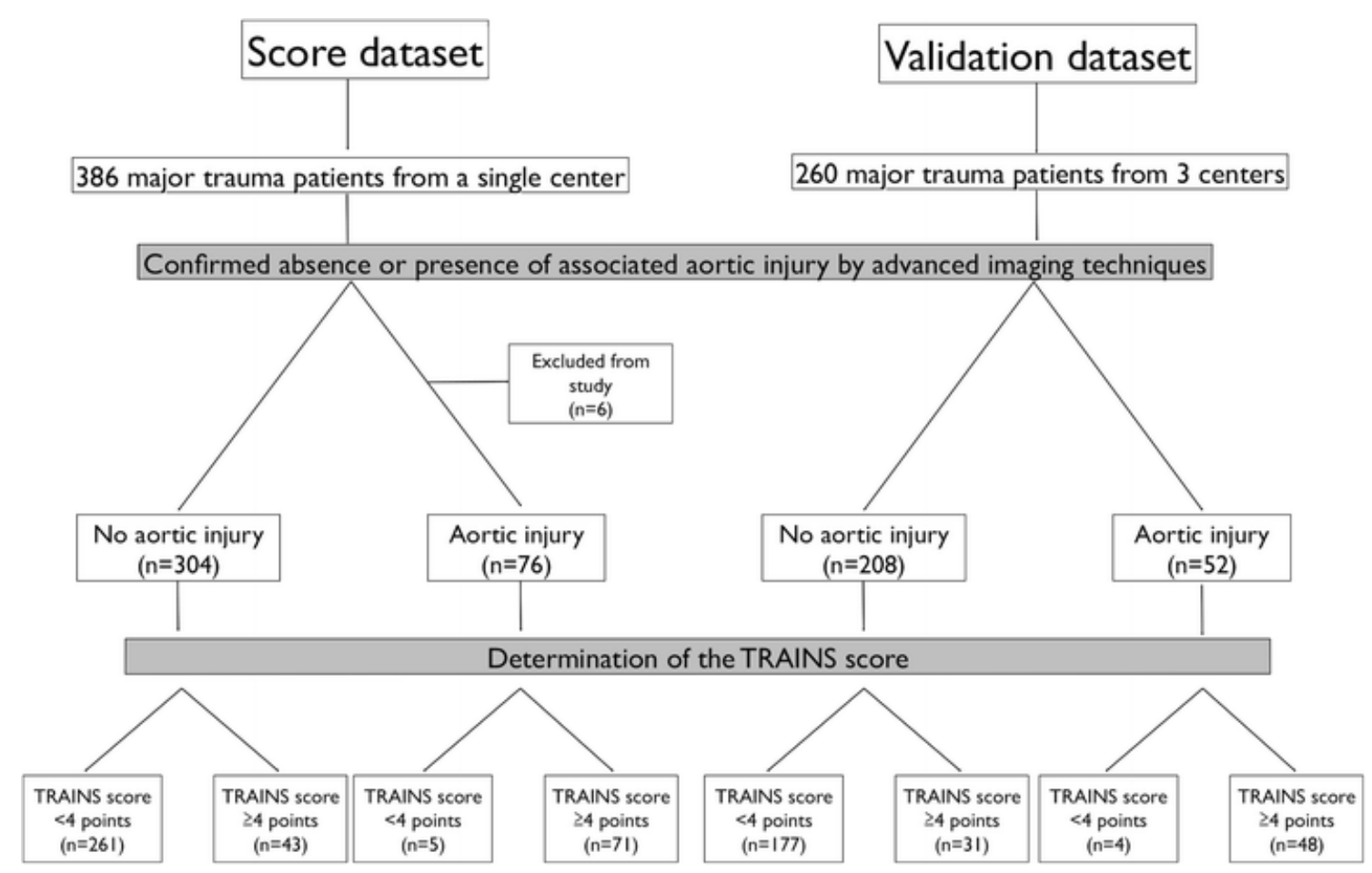

Fig. 1. Study design for recruitment of both the score and validation data sets. Exclusion criteria were penetrating trauma, deficient documentation, and/or in extremis status on arrival. TRAINS traumatic aortic injury score 
Acute traumatic aortic injury diagnosis was based on imaging CT scan, angiography, and/or transesophageal echocardiogram (TEE).

All participating centers used the same CT scan acquisition protocols for trauma patients requiring advanced imaging from January 2000.

All institutions received institutional review board (IRB) approval to participate in the study; each IRB waived the requirement for written patient consent.

\section{Variables collected}

Data on 96 variables were recorded on a standardized form that included information on patient demographics, mechanism of injury, clinical status on hospital admission (blood pressure, respiratory rate, need of endotracheal intubation at the site of the trauma or during transport, Glasgow coma scale (GCS)), injury severity score (ISS) [9], abbreviated injury score (AIS) for each body area (head, chest, abdomen, extremities), revised trauma score (RTS) [11], trauma injury severity score (TRISS) [12], associated injuries, findings in simple CXR taken on admission and other performed diagnostic imaging tests (CT scan, angiography, TEE).

When present, aortic injury was classified according to its severity, i.e., type I (intimal tear), type II (intramural hematoma), type III (pseudoaneurysm), or type IV (rupture) [13], and the site of injury was also recorded.

Mechanism of injury was classified as motor vehicle crash (MVC), motorcycle collision (MCC), auto versus pedestrian (AVP), fall, crush under weight, and others.

For the purpose of the study, the definitions of other analyzed variables, i.e., widened mediastinum [14-17], hemothorax [18], lung contusion [19], pelvic fracture-deformity [20], long bong fracture, left scapula fracture [21], hypotension, abnormal respiratory rate, and head injury, are included as Online Resource 1.

\section{Statistical analysis}

Data are expressed as mean and standard deviation or median and range, when appropriate. For bivariate analysis, proportions were compared with contingency tables by means of chi-square or Fisher exact tests and the Student's $t$ test or Mann-Whitney rank-sum test was used to compare continuous variables.

The relation between the severity of the trauma, defined by the TRISS, RTS, and ISS values, and the different degrees of aortic injury was tested using one-way analysis of variance (ANOVA). One-way ANOVA was also used to determine whether there was association between the TRAINS value and the degree of severity of the ATAIs.

A bivariate analysis was used to identify variables potentially influencing the probability of presenting with an ATAI among major trauma patients. A stepwise forward logistic regression was used to confirm or reject these clinically relevant variables as predictors of aortic injury. Odds ratio (OR), $95 \% \mathrm{CI}$, and $p$ values were derived. A $p$ value of less than 0.05 was considered significant.

Subsequently, a prediction score to determine the probability of aortic injury from clinical and CXR data was developed. Predictive variables confirmed by logistic regression were assigned a score according to their corresponding beta coefficient (provided by logistic regression), which was rounded to the closest integer value (1-4).

The receiver operating characteristic (ROC) curve and the Hosmer-Lemeshow goodness-of-fit statistic were calculated to assess the performance and calibration of the model. The DeLong method [22] was employed to compare areas under ROC curves.

The Youden index was used to measure the effectiveness of the test to select an optimal threshold value (cutoff point) for the test [23].

We performed both an internal and external-multicenter validation of the score. Internal validation of the aortic injury predictive score was accomplished using the bootstrap technique.

The study adheres to the standards for reporting of diagnostic accuracy (STARD) initiative [24].

The SPSS statistical program for windows version 17.0 (SPSS, Chicago, IL) was used to perform data analysis.

A more extensive description of the statistical methods is included as Online Resource 2. 


\section{Results}

Clinical and radiological data were available for all the patients in the score data set (Table 1).

Table 1. Epidemiological, clinical, and diagnostic characteristics of patients in the score data set

\begin{tabular}{|c|c|c|c|}
\hline Variable & ATAI group & NATAI group & $p$ value \\
\hline Sex (male) & $82.9 \%$ & $85.2 \%$ & 0.61 \\
\hline Age & $41.33 \pm 18.14$ & $43.62 \pm 18.30$ & 0.32 \\
\hline Age $\geq 55$ (years) & $26.3 \%$ & $29.3 \%$ & 0.61 \\
\hline \multicolumn{4}{|l|}{ Mechanism of injury } \\
\hline MVC & $61.8 \%$ & $39.2 \%$ & \multirow{6}{*}{0.002} \\
\hline $\mathrm{MCC}$ & $14.5 \%$ & $14.8 \%$ & \\
\hline Fall & $10.5 \%$ & $26 \%$ & \\
\hline AVP & $6.6 \%$ & $11.5 \%$ & \\
\hline Crush under weight & $5.3 \%$ & $7.2 \%$ & \\
\hline Others & $1.3 \%$ & $1.3 \%$ & \\
\hline \multicolumn{4}{|l|}{ Diagnostic tests on admission } \\
\hline CT scan & $68.4 \%$ & $91.7 \%$ & $<0.001$ \\
\hline Angiography & $42.1 \%$ & $12.2 \%$ & $<0.001$ \\
\hline TEE & $63.1 \%$ & $26.9 \%$ & $<0.001$ \\
\hline ISS & $40.45 \pm 14.32$ & $29.95 \pm 11.03$ & $<0.001$ \\
\hline RTS & $5.98 \pm 1.71$ & $6.97 \pm 1.34$ & $<0.001$ \\
\hline TRISS & $38.06 \pm 36.44$ & $18.54 \pm 24.91$ & $<0.001$ \\
\hline \multicolumn{4}{|l|}{ Type of aortic injury } \\
\hline Type I (intimal tear) & $25 \%$ & \multirow{4}{*}{ NA } & \multirow{4}{*}{ NA } \\
\hline Type II (intramural hematoma) & $22.4 \%$ & & \\
\hline Type III (pseudoaneurysm) & $22.4 \%$ & & \\
\hline Type IV (rupture) & $30.2 \%$ & & \\
\hline \multicolumn{4}{|l|}{ Location of aortic injury } \\
\hline Aortic isthmus & $64.5 \%$ & \multirow{4}{*}{ NA } & \multirow{4}{*}{ NA } \\
\hline Mid-distal descending aorta & $19.7 \%$ & & \\
\hline Aortic arch & $11.9 \%$ & & \\
\hline Ascending aorta & $3.9 \%$ & & \\
\hline
\end{tabular}

The $p$ value of proportions analysis was obtained with the $\chi^{2}$ test, whereas $p$ value mean analysis corresponds to Student's $t$ test $A T A I$ acute traumatic aortic injury, NATAI no associated acute traumatic aortic injury, $M V C$ motor vehicle crash, $M C C$ motorcycle collision, $A V P$ auto versus pedestrian, $C T$ computed tomography, TEE trans-esophageal echocardiography, ISS injury severity score, AIS abbreviated injury score, RTS revised trauma score, TRISS trauma injury severity score, NA not applicable

Bivariate analysis suggested 18 variables potentially influencing the probability of presenting with an aortic injury in major trauma patients (Table 2). Eleven other analyzed variables were not statistically significant in bivariate analysis (Table 2). 
Table 2. Results of the univariate analysis for the patients in the score data set

\begin{tabular}{|c|c|c|c|}
\hline First rib fracture & 17.1 & 8.2 & 0.021 \\
\hline Right ribs fracture & 31.6 & 43.4 & 0.061 \\
\hline Sternal fracture & 9.2 & 5.9 & 0.301 \\
\hline Left clavicle fracture & 11.8 & 8.6 & 0.375 \\
\hline Left scapula fracture & 28.9 & 7.9 & $<0.001$ \\
\hline Right scapula fracture & 2.6 & 5.6 & 0.290 \\
\hline Pelvic fracture & 51.3 & 15.5 & $<0.001$ \\
\hline Long bone fracture & 21.1 & 4.6 & $<0.001$ \\
\hline Head injury & 21.1 & 18.7 & 0.61 \\
\hline Spine fracture & 24.3 & 23.7 & 0.905 \\
\hline Spleen injury & 21.1 & 14.5 & 0.159 \\
\hline Bowel injury & 10.5 & 5.3 & 0.092 \\
\hline Kidney injury & 18.4 & 7.9 & 0.006 \\
\hline Bladder injury & 3.9 & 0.7 & 0.024 \\
\hline Hemoperitoneum & 43.4 & 17.8 & $<0.001$ \\
\hline Pneumoperitoneum & 1.3 & 3.3 & 0.359 \\
\hline Hemothorax & 77.6 & 44.7 & $<0.001$ \\
\hline Pneumothorax & 38.2 & 39.5 & 0.834 \\
\hline Widened mediastinum & 78.9 & 24 & $<0.001$ \\
\hline Hypotension & 76.3 & 19.1 & $<0.001$ \\
\hline Altered respiratory rate & 59.2 & 30.6 & $<0.001$ \\
\hline
\end{tabular}

ATAI acute traumatic aortic injury, NATAI no associated acute traumatic aortic injury, ETI endotracheal intubation, GCS Glasgow coma score

Of the 18 potentially influencing variables suggested by the bivariate analysis, the stepwise forward logistic regression only confirmed seven variables as risk factors for the presence of associated traumatic aortic injury in major trauma patients (Table 3). These variables were assigned a score between 1 and 4 points according to their corresponding beta coefficient provided by logistic regression, which was rounded to the closest integer value (1-4), as shown in Table 3 . Thus, the obtained score could rank from 0 to 12 points.

Table 3. Results of the binary stepwise forward logistic regression and the corresponding score assigned to each significant variable according to its OR

\begin{tabular}{|c|c|c|c|c|c|}
\hline Widened mediastinum & 3.42 & 30.82 & $12.05-78.81$ & $<0.001$ & 4 \\
\hline Long bone fracture & 2.15 & 8.60 & $2.15-34.31$ & 0.002 & 2 \\
\hline Lung contusion & 1.41 & 4.12 & $1.11-15.20$ & 0.033 & 1 \\
\hline Left scapula fracture & 1.34 & 3.81 & $1.24-11.69$ & 0.019 & 1 \\
\hline Pelvic fracture-deformity & 1.08 & 2.96 & $1.15-7.60$ & 0.024 & 1 \\
\hline
\end{tabular}

$O R$ odds ratio, $C I$ confidence interval 
The ROC curve had an area under the curve of 0.96 (0.94-0.98) (Fig. 2). There was no statistically significant difference by the DeLong method between this ROC curve and the ROC curve obtained using the non-rounded original beta coefficients, which had an area under the curve of $0.97(0.95-0.98)$ $(p<0.001)$ (Fig. 2).

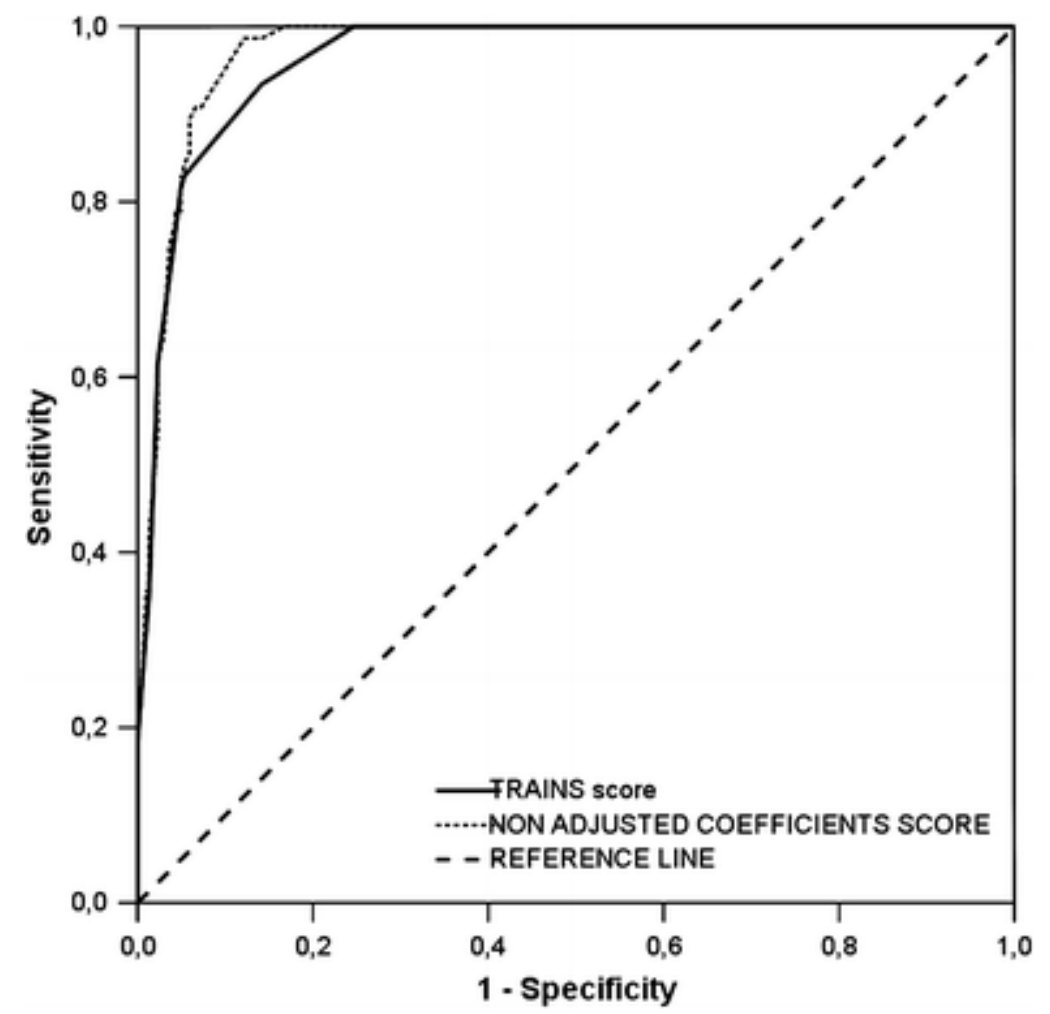

Fig. 2. ROC curve in the score data set had an area under the curve of $0.96(0.94-0.98)$. There was no statistically significant difference by the DeLong method between this ROC curve and the ROC curve obtained using the non-rounded original beta coefficients, which had an area under the curve of $0.97(0.95-0.98)(p<0.001)$

The Hosmer-Lemeshow goodness-of-fit statistic across groups of risk was not statistically significant ( $p=0.07$, Online Resource 3), indicating little departure from a perfect fit.

A score of at least 4 points was calculated as the threshold value which maximized sensitivity and specificity. It provided a sensitivity of 93.42\% (87.19-99.65) and a specificity of $85.85 \%$ (81.77-89.94). The Youden's index for a score of at least 4 was $0.79(0.72-0.86)$, whereas the positive likelihood ratio was 6.60 (4.98-8.77) and negative likelihood ratio was $0.08(0.03-0.18)$.

Other tested cutoff values were a score of at least 3 points, which provided a sensitivity of $97.2 \%$ (95.2-100) and a specificity of $53 \%(47.34-58.65)$, and a score of at least 5 points, which had a sensitivity of $74.68 \%(64.46-84.9)$ and a specificity of $94.74 \%(92.06-97.41)$.

One-way ANOVA revealed that there was no relation between the severity of the trauma defined by either TRISS $(p=0.77)$, ISS $(p=0.59)$, or RTS $(p=0.73)$ values and severity of aortic injury (types I to IV). Nonetheless, the ANOVA test demonstrated that there was a significant relation between TRAINS value and severity of aortic injury $(p=0.005)$. Additional data are given as Online Resource 4.

Data for the patients of the validation data set are shown in Table 4. 
Table 4. Epidemiological, clinical, and diagnostic characteristics of patients in the validation data set

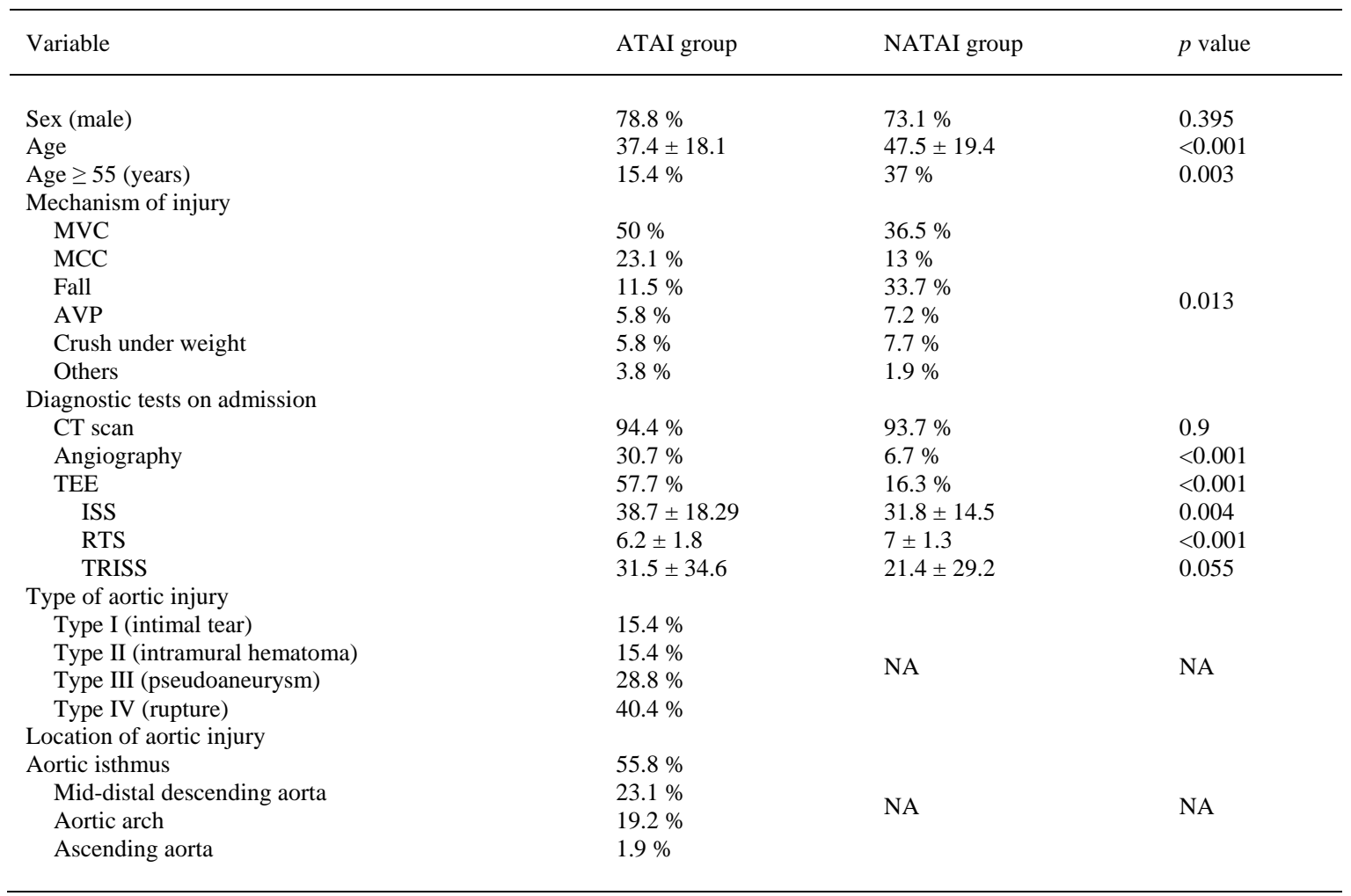

The $p$ value of proportions analysis was obtained with the $\chi^{2}$ test, whereas $p$ value mean analysis corresponds to Student's $t$ test ATAI acute traumatic aortic injury, NATAI no associated acute traumatic aortic injury, $M V C$ motor vehicle crash, $M C C$ motorcycle collision, $A V P$ auto versus pedestrian, $C T$ computed tomography, TEE trans-esophageal echocardiography, ISS injury severity score, $A I S$ abbreviated injury score, $R T S$ revised trauma score, TRISS trauma injury severity score, NA not applicable

In the validation data set, the score provided a sensitivity of $92.31 \%(86.1-100)$ and a specificity of $85.1 \%$ (80.02-90.18). The Youden's index for a score of at least 4 was $0.77(0.69-0.86)$, whereas the positive likelihood ratio was 6.19 (4.43-8.65) and negative likelihood ratio was 0.09 (0.04-0.23).

The one-way ANOVA also confirmed that there was a significant relation between TRAINS value and severity of aortic injury $(p=0.002)$ in the validation data set.

\section{Discussion}

This research presents for the first time in the literature a predictive scoring method for ATAIs in major trauma patients with associated blunt chest trauma. The method, which was externally validated in a multicenter study, is based on simple variables easy to obtain in the emergency room and has remarkable proven sensitivity and specificity.

The score and the associated algorithm were designed to rapidly identify major trauma patients at high risk of suffering an ATAI and to provide a framework to optimize resources use and to initiate the prompt medical management to prevent potentially lethal aortic-related complications.

In daily practice, CXR on admission is used to provide data to guide suspicion of ATAIs in major trauma patients. A widened mediastinum [17] and variations such as a left mediastinal width of $6 \mathrm{~cm}$ or more and a mediastinal width ratio of at least 0.60 [25] and other CXR findings [26, 27] are frequently associated with the diagnosis of an ATAI and used in the decision to proceed to more advanced imaging tests. Nonetheless, although combining the most sensitive radiographic signs may improve sensitivity up to $90 \%$ in certain series, there is a simultaneous decrease in specificity (even <50\%) which fails to provide a sufficient negative predictive value [17]. In addition, it has been reported in the literature that up to $30 \%$ of patients with ATAIs may not present mediastinal abnormalities [27]. The vast majority of major trauma patients (97.9\% of the patients in our study) had a CXR taken in the supine position using portable imaging equipment. Thus, in a significant number of cases, the interpretation of CXR findings in 
major trauma patients may be difficult because of the poorer technical quality of supine radiographs taken using portable equipment $[15,17]$.

We developed a highly predictive but easy scoring method based on clinical and CXR data with a sensitivity of $93.42 \%(87.19-99.65)$ and a specificity of $85.85 \%$ (81.77-89.94) in our center's population (score data set) and with a sensitivity of $92.31 \%(86.1-100)$ and a specificity of $85.1 \%$ (80.02-90.18) after an independent external multicenter validation process (validation data set). The process of external validation is of paramount importance to check the validity of the model across other geographic areas [28].

In order to allow the prompt identification of major trauma patients at risk of suffering a potentially lethal aortic injury, we currently recommend in all patients with a TRAINS of at least 4 to initiate an optimal medical control [3, 4] and we advocate for performing a specific aortic MDCT protocol combined with a TEE, especially in unstable and/or intubated patients [7]. The speed and portability of TEE, combined with its ability to obtain high-resolution images of the aorta make this technique an attractive diagnostic modality, especially in an unstable patient in whom it can be performed without interrupting ongoing measures to stabilize the patient $[29,30]$.

In addition, patients with a score of at least 4 (high risk of ATAI) should undergo a three-phase vascular MDCT including an unenhanced phase, an arterial contrast-enhanced phase from the thoracic inlet to the symphysis pubis, and a delayed phase. Whenever the score is at least 4 , it is mandatory to generate oblique reconstructions, resembling the images obtained in conventional angiography, as well as sagittal, coronal, and MPR [31]. In such cases, we recommend to perform an MDCT using $100 \mathrm{~mL}$ of intravenous iodinated contrast medium at $4 \mathrm{~mL} / \mathrm{s}$ to maximize arterial enhancement, acquisition of axial images at $0.625 \mathrm{~mm}$ collimation, and reviewing images at a section thickness of $5 \mathrm{~mm}$.

In contrast, patients with a score of less than 4 (low risk of ATAI) are managed with simple CXRs, data from extended focused assessment with sonography for trauma (eFAST) [32], and, when indicated because of a suspected non-aortic thoracic injury, a thoracic or thoraco-abdominopelvic less aggressive protocol of two phases MDCT. In that protocol, axial images are acquired at $1.25 \mathrm{~mm}$ collimation during the portal venous phase, after injection of $80 \mathrm{~mL}$ of iodinated contrast medium at $2 \mathrm{~mL} / \mathrm{s}$. This approach minimizes the contrast and radiation exposure of the patient compared to a three-phase vascular MDCT.

The use of a standard trauma (nonspecific arterial) MDCT scan protocol without MPR allows many high-degree ATAIs to be diagnosed, but up to $10 \%$ of less severe aortic injuries [33] can be missed. Although low-degree aortic injuries usually do not pose a life-threatening risk at the moment of trauma admission, their long-term natural history is not well known and may lead to potential adverse consequences $[13,33,34]$.

Our current algorithm for managing major trauma patients with associated blunt chest trauma is depicted in Fig. 3. 


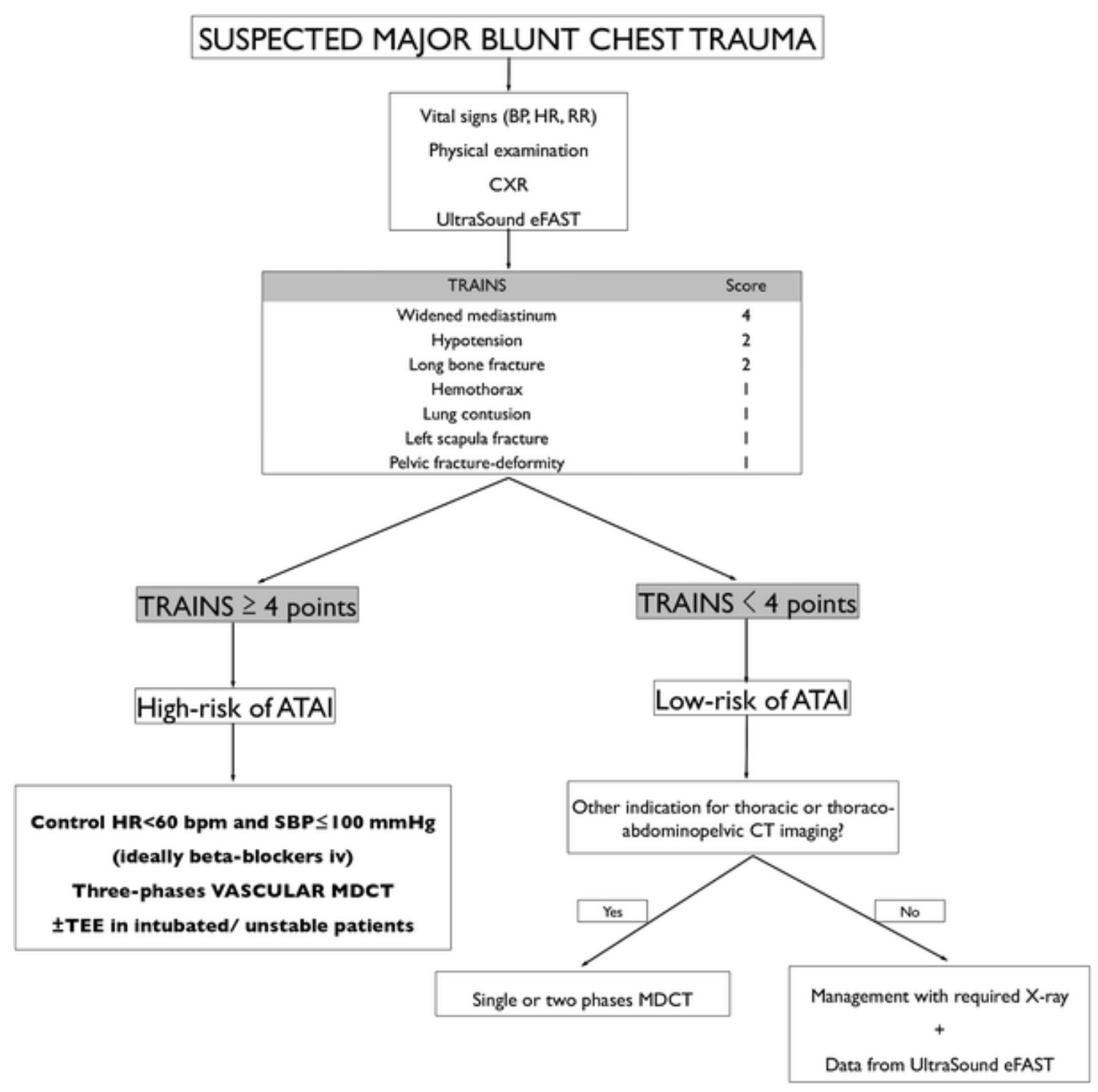

Fig. 3. Proposed algorithm for managing major trauma patients with associated blunt chest trauma according also to the international recommendations for advance imaging test $[2,8]$ and medical therapy $[3,4]$ in patients at high risk of ATAI. BP blood pressure, $H R$ heart rhythm, $R R$ respiratory rate, $C X R$ chest X-ray, $e F A S T$ extended focused assessment with sonography for trauma, $M D C T$ multidetector computed tomography

Other alternative cutoff values such as a score of at least 5 points were rejected because, despite providing a higher specificity $(94.74 \%)$, the decrease of sensitivity (74.68\%) would probably lead to missed diagnosis of a significant number of traumatic aortic injuries, which is not affordable from a clinical point of view. Conversely, a cutoff value of at least 3 points with a higher sensitivity $(97.2 \%)$ had an unacceptably low specificity (53\%), which would involve an overutilization of advanced thoracic imaging tests in an important number of patients without aortic injury (false positives). Thus, a score with a low specificity might entail an unnecessary increase in hospital resources and financial costs and, moreover, an unnecessary deleterious exposure to nephrotoxic contrast and radiation.

As other authors have found [33], the conventional trauma risk scores (ISS, RTS, and TRISS) failed to show a statistical relationship between the severity of the trauma and the degree of severity of the aortic injury. Thus, the conventional trauma severity scores are useless in raising diagnostic suspicion of ATAI. In contrast, the TRAINS value was proven to be related to the severity of the aortic injury in both data sets. In fact, the greater the TRAINS value was, the more severe the aortic injury was.

Apart from the previously mentioned CXR findings suggestive of ATAI, clinically relevant correlations between non-mediastinal injuries and ATAI have been reported [16, 35-37]. 
Blackmore et al. [38] published a traumatic aortic injury prediction rule based on a single-center retrospective case-control study. Although innovative, the number of cases was very low. Besides, that study lacked an external validation in other populations to ensure generalizability. In fact, a more recent re-evaluation of those clinical predictors by Kirkham and Blackmore [39] showed that only four factors were actually predictive [39].

To the best of our knowledge, TRAINS is the first predictive score of ATAI in major trauma patients externally validated in a multicenter study.

\section{Limitations}

There are limitations with our model that need to be considered, including the limitations inherent in any retrospective study. The score data set was obtained from a long time period during which substantial diagnostic and therapeutic advances were incorporated. The applicability of the score is limited to major trauma patients (ISS > 15) with associated chest trauma. The rounding of the variable "widened mediastinum" to 4 points might jeopardize the score from a pure statistical point of view, but this modification improves the applicability of the score in the everyday clinical practice.

The simplicity of the scoring method, the fact that it does not depend on the result of complex diagnostic tests, and its validation in a contemporaneous multicenter population overcome these shortcomings. An extended comment of the limitations of the study is given as Online Resource 5.

\section{Conclusions}

We have developed a multivariate prediction model for traumatic aortic injury after major trauma with associated blunt chest trauma. TRAINS may be used in daily practice to easily and rapidly identify major trauma patients with associated blunt chest trauma at risk of aortic injury, thus avoiding unnecessary cost and radiation exposure in low-risk trauma patients. This tool may also be useful for planning of resource allocation, enabling clinicians to refer patients at high risk of traumatic aortic injuries to specialized units and providing the chance of a prompt specific diagnostic and therapeutic management of this critical subset of trauma patients to avoid potentially lethal aortic-related complications. TRAINS is able to raise suspicion of ATAI even in trauma cases with low-degree aortic injuries, thus recommending the performance of a specific arterial MDCT scan with MPR and avoiding the misdiagnosis of aortic injuries.

\section{References}

1. Smith RS, Chang FC (1986) Traumatic rupture of the aorta: still a lethal injury. Am J Surg 152:660-663

2. Steenburg SD, Ravenel JG (2008) Acute traumatic thoracic aortic injuries: experience with 64-MDCT. Am J Roentgenol 191:1564-1569

3. Fabian TC, Davis KA, Gavant ML, Croce MA, Melton SM, Patton JH Jr, Haan CK, Weiman DS, Pate JW (1998) Prospective study of blunt aortic injury: helical CT is diagnostic and antihypertensive therapy reduces rupture. Ann Surg 227:666-676 discussion 676-667

4. Tsai TT, Nienaber CA, Eagle KA (2005) Acute aortic syndromes. Circulation 112:3802-3813

5. Demetriades D, Velmahos GC, Scalea TM, Jurkovich GJ, Karmy-Jones R, Teixeira PG, Hemmila MR, O'Connor JV, McKenney MO, Moore FO, London J, Singh MJ, Lineen E, Spaniolas K, Keel M, Sugrue M, Wahl WL, Hill J, Wall MJ, Moore EE, Margulies D, Malka V, Chan LS (2008) Operative repair or endovascular stent graft in blunt traumatic thoracic aortic injuries: results of an American Association for the Surgery of Trauma multicenter study. J Trauma 64:561-570, discussion 570-561

6. Demetriades D, Velmahos GC, Scalea TM, Jurkovich GJ, Karmy-Jones R, Teixeira PG, Hemmila MR, O'Connor JV, McKenney MO, Moore FO, London J, Singh MJ, Spaniolas K, Keel M, Sugrue M, Wahl WL, Hill J, Wall MJ, Moore EE, Lineen E, Margulies D, Malka V, Chan LS (2009) Blunt traumatic thoracic aortic injuries: early or delayed repair - results of an American Association for the Surgery of Trauma prospective study. J Trauma 66:967-973

7. Mosquera VX, Marini M, Lopez-Perez JM, Muniz-Garcia J, Herrera JM, Cao I, Cuenca JJ (2011) Role of conservative management in traumatic aortic injury: comparison of long-term results of conservative, surgical, and endovascular treatment. J Thorac Cardiovasc Surg 142:614-621

8. Mirvis SE, Shanmuganathan K (2007) Diagnosis of blunt traumatic aortic injury 2007: still a nemesis. Eur J Radiol 64:27-40

9. Baker SP, O’Neill B, Haddon W Jr, Long WB (1974) The injury severity score: a method for describing patients with multiple injuries and evaluating emergency care. J Trauma 14:187-196

10. Soreide K (2009) Epidemiology of major trauma. Br J Surg 96:697-698

11. Champion HR, Sacco WJ, Copes WS, Gann DS, Gennarelli TA, Flanagan ME (1989) A revision of the Trauma Score. J Trauma 29:623-629 
12. Champion HR, Sacco WJ, Copes WS (1995) Injury severity scoring again. J Trauma 38:94-95

13. Lee WA, Matsumura JS, Mitchell RS, Farber MA, Greenberg RK, Azizzadeh A, Murad MH, Fairman RM (2011) Endovascular repair of traumatic thoracic aortic injury: clinical practice guidelines of the Society for Vascular Surgery. J Vasc Surg 53(1):187-192

14. Marnocha KE, Maglinte DD, Woods J, Goodman M, Peterson P (1984) Mediastinal-width/chest-width ratio in blunt chest trauma: a reappraisal. Am J Roentgenol 142:275-277

15. Woodring JH, Dillon ML (1984) Radiographic manifestations of mediastinal haemorrhage from blunt chest trauma. Ann Thorac Surg 37:171-178

16. Fabian TC, Richardson JD, Croce MA, Smith JS Jr, Rodman G Jr, Kearney PA, Flynn W, Ney AL, Cone JB, Luchette FA, Wisner DH, Scholten DJ, Beaver BL, Conn AK, Coscia R, Hoyt DB, Morris JA Jr, Harviel JD, Peitzman AB, Bynoe RP, Diamond DL, Wall M, Gates JD, Asensio JA, Enderson BL et al (1997) Prospective study of blunt aortic injury: multicenter trial of the American Association for the Surgery of Trauma. J Trauma 42:374-380, discussion 380-373

17. Cook AD, Klein JS, Rogers FB, Osler TM, Shackford SR (2001) Chest radiographs of limited utility in the diagnosis of blunt traumatic aortic laceration. J Trauma 50:843-847

18. Mowery NT, Gunter OL, Collier BR, Diaz JJ Jr, Haut E, Hildreth A, Holevar M, Mayberry J, Streib E (2011) Practice management guidelines for management of hemothorax and occult pneumothorax. J Trauma 70:510-518

19. Cohn SM, Dubose JJ (2010) Pulmonary contusion: an update on recent advances in clinical management. World J Surg 34:1959-1970

20. Lee C, Porter K (2007) The pre-hospital management of pelvic fractures. Emerg Med J 24:130-133

21. Cole PA (2002) Scapula fractures. Orthop Clin North Am 33:1-18 vii

22. DeLong ER, DeLong DM, Clarke-Pearson DL (1988) Comparing the areas under two or more correlated receiver operating characteristic curves: a nonparametric approach. Biometrics 44:837-845

23. Fluss R, Faraggi D, Reiser B (2005) Estimation of the Youden index and its associated cut-off point. Biom J 47:458-472

24. Bossuyt PM, Reitsma JB, Bruns DE, Gatsonis CA, Glasziou PP, Irwig LM, Lijmer JG, Moher D, Rennie D, de Vet HC (2003) Towards complete and accurate reporting of studies of diagnostic accuracy: the STARD initiative. BMJ 326:41-44

25. Wong YC, Ng CJ, Wang LJ, Hsu KH, Chen CJ (2004) Left mediastinal width and mediastinal width ratio are better radiographic criteria than general mediastinal width for predicting blunt aortic injury. J Trauma 57:88-94

26. Mirvis SE, Bidwell JK, Buddemeyer EU, Diaconis JN, Pais SO, Whitley JE (1987) Imaging diagnosis of traumatic aortic rupture. A review and experience at a major trauma center. Invest Radiol 22:187-196

27. Woodring JH (1990) The normal mediastinum in blunt traumatic rupture of the thoracic aorta and brachiocephalic arteries. J Emerg Med 8:467-476

28. Laupacis A, Sekar N, Stiell IG (1997) Clinical prediction rules. A review and suggested modifications of methodological standards. JAMA 277:488-494

29. Smith MD, Cassidy JM, Souther S, Morris EJ, Sapin PM, Johnson SB, Kearney PA (1995) Trans-esophageal echocardiography in the diagnosis of traumatic rupture of the aorta. N Engl J Med 332:356-362

30. Catoire P, Orliaguet G, Liu N, Delaunay L, Guerrini P, Beydon L, Bonnet F (1995) Systematic trans-esophageal echocardiography for detection of mediastinal lesions in patients with multiple injuries. J Trauma 38:96-102

31. Berger FH, van Lienden KP, Smithuis R, Nicolaou S, van Delden OM (2010) Acute aortic syndrome and blunt traumatic aortic injury: pictorial review of MDCT imaging. Eur J Radiol 74:24-39

32. Xirouchaki N, Magkanas E, Vaporidi K, Kondili E, Plataki M, Patrianakos A, Akoumianaki E, Georgopoulos D (2011) Lung ultrasound in critically ill patients: comparison with bedside chest radiography. Intensive Care Med 37:1488-1493

33. Malhotra AK, Fabian TC, Croce MA, Weiman DS, Gavant ML, Pate JW (2001) Minimal aortic injury: a lesion associated with advancing diagnostic techniques. J Trauma 51:1042-1048

34. Kepros J, Angood P, Jaffe CC, Rabinovici R (2002) Aortic intimal injuries from blunt trauma: resolution profile in nonoperative management. J Trauma 52:475-478

35. Cook J, Salerno C, Krishnadasan B, Nicholls S, Meissner M, Karmy-Jones R (2006) The effect of changing presentation and management on the outcome of blunt rupture of the thoracic aorta. J Thorac Cardiovasc Surg 131:594-600

36. Burkhart HM, Gomez GA, Jacobson LE, Pless JE, Broadie TA (2001) Fatal blunt aortic injuries: a review of 242 autopsy cases. J Trauma 50:113-115

37. Teixeira PG, Inaba K, Barmparas G, Georgiou C, Toms C, Noguchi TT, Rogers C, Sathyavagiswaran L, Demetriades D (2011) Blunt thoracic aortic injuries: an autopsy study. J Trauma 70:197-202

38. Blackmore CC, Zweibel A, Mann FA (2000) Determining risk of traumatic aortic injury: how to optimize imaging strategy. Am J Roentgenol 174:343-347

39. Kirkham JR, Blackmore CC (2007) Screening for aortic injury with chest radiography and clinical factors. Emerg Radiol 14:211-217 